\title{
Tumor microenvironment immune types in gastric cancer are associated with mismatch repair however, not HER2 status
}

\author{
ANNA MARIA VALENTINI $^{1}$, FEDERICA DI PINTO ${ }^{1}$, SERGIO COLETTA ${ }^{1}$, \\ VITO GUERRA $^{2}$, RAFFAELE ARMENTANO ${ }^{1}$ and MARIA LUCIA CARUSO ${ }^{1}$ \\ Departments of ${ }^{1}$ Pathology and ${ }^{2}$ Epidemiology, National Institute of \\ Gastroenterology ‘S. de Bellis', Research Hospital, Castellana Grotte, I-70013 Bari, Italy
}

Received August 22, 2018; Accepted February 18, 2019

DOI: $10.3892 / \mathrm{ol} .2019 .10513$

\begin{abstract}
The treatment of patients with human epidermal growth factor receptor 2 (HER2)-negative gastric cancer is a major challenge. Immunotherapy using immune checkpoint inhibitors is a rapidly growing field. In a number of malignancy types it has been demonstrated that patients with mismatch repair deficiency efficiently respond to programmed death-ligand 1 (PD-L1) blockade therapy. Recent studies have evaluated tumor microenvironment immune types to predict which patients may clinically benefit from immunotherapy. The present study aimed to evaluate the immunohistochemical expression of PD-L1 in 70 gastric cancer tissue samples. Potential associations between PD-L1 expression and mismatch repair deficiency, HER2 and Epstein Barr virus (EBV) status were then investigated in the context of the tumor microenvironment. A positive association was identified for PD-L1 expression with mismatch repair deficiency and EBV status; however, no association was revealed with HER2 status. Immunohistochemistry was then used to classify the microenvironment immune types. This demonstrated that the majority of the gastric cancer samples (73\%) belonged to the tumor microenvironment immune type II [PD-L1\%/cluster of differentiation $8(\mathrm{CD} 8)^{+}$low], which involves an immune ignorant state and has a low sensitivity to immunotherapy. However,
\end{abstract}

Correspondence to: Dr Anna Maria Valentini, Department of Pathology, National Institute of Gastroenterology 'S. de Bellis', Research Hospital, Via Turi 27, Castellana Grotte, I-70013 Bari, Italy E-mail: am.valentini@irccsdebellis.it

Abbreviations: GC, gastric carcinoma; IHC, immunohistochemistry; IIC, infiltrating immune cell; MMR, mismatch repair; MMR-D, mismatch repair deficiency; MMR-P, mismatch repair proficient; MSI, microsatellite instability; PD-1, programmed cell death protein-1; PD-L1, programmed death-ligand 1; TC, tumor cell; TIL, tumor infiltrating lymphocyte; TMIT, tumor microenvironment immune type

Key words: programmed death-ligand 1, immune checkpoints, immunotherapy, mismatch repair deficiency, tumor microenvironment immune type
$7 \%$ of the gastric cancer cases were identified to belong to the tumor microenvironment immune type I (PD-L1 ${ }^{+} / \mathrm{CD}^{+}$high), which exhibits adaptive immune escape responses and a high chance of reversion with immune checkpoint blockade therapy. In conclusion, the present study emphasized the importance of evaluating tumor microenvironment immune types, mismatch repair deficiency status and EBV status, rather than PD-L1 expression alone, when evaluating the eligibility of a patient for immunotherapy with anti-programmed cell death protein-1/PD-L1 antibodies.

\section{Introduction}

Although the incidence rate of gastric cancer (GC) has decreased $\sim 3 \%$ per year in the last few decades, GC has been reported as the second leading cause of cancer-associated mortality worldwide, behind lung cancer in 2012 (1). Unfortunately, the majority of GC cases are diagnosed at an advanced stage; therefore, the clinical outcome of patients with GC remains poor and the 5-year survival rate of patients with advanced metastatic disease is $<30 \%$ (2). Therefore, the most effective treatment strategies for patients with advanced GC include chemotherapy and novel targeted therapies (2). Unlike other tumor types that have a number of therapeutic options based on the molecular characteristic of the tumor, the genomic landscape of GC is highly heterogeneous (3). Therefore, it can be difficult to target the entire GC tumor as sub-clones of GC cells possess different biological behaviors (4). In addition to Trastuzumab, an anti-human epidermal growth factor receptor 2 (HER2) antibody, and Ramucirumab, an anti-vascular endothelial growth factor receptor antibody (5), the Food and Drugs Administration (FDA) has recently approved Pembrolizumab, an anti-programmed cell death protein-1 (PD-1) antibody, for the treatment of patients with metastatic gastric cancer and high PD-L1 immunohistochemical expression $(6,7)$. Immunotherapy has revolutionized cancer treatment by introducing new therapies, including checkpoint inhibitors that target the host immune system instead of tumor cells (8).

The PD-1 and programmed death-ligand 1 (PD-L1) pathway is one of the most widely studied immune checkpoint pathways as it is crucial in physiological conditions for the maintenance of self-tolerance and preventing 
autoimmune diseases (9), and for evading antitumor immunity (10). PD-1 is an immune-inhibitory receptor that is constitutively expressed by activated $\mathrm{T}$ lymphocytes and macrophages, whereas other non-T lymphocytes, including B cells and natural killer cells, only express PD-1 following cytokine-induced stimulation $(11,12)$. In addition to being constitutively expressed by T cells, B cells, macrophages and dendritic cells, PD-L1 is also expressed on tumor cells (TCs) in several types of cancer. The interaction between PD-1 on $\mathrm{T}$ cells and PD-L1 on TCs inhibits the cluster of differentiation $8(\mathrm{CD} 8)^{+} \mathrm{T}$ cell-mediated immune response, which induces an immunosuppressive microenvironment within the tumor and promotes evasion of immune destruction (12). PD-1/PD-L1 inhibitors can restore antitumor immunity and immunotherapy using immune checkpoint inhibitors has been reported to be effective in certain cancer types, including malignant melanoma (13) and non-small cell lung cancer (14). However, the sensitivity to Pembrolizumab may not be the same in all patients eligible for immunotherapy; therefore, it may be useful to identify adjunctive biomarkers associated with the expression of PD-L1, which may allow a more accurate and targeted selection of eligible patients.

In colorectal cancer, it has been demonstrated that the majority of patients with mismatch repair deficiency (MMR-D) are good responders to anti-PD-1/PD-L1 therapy $(15,16)$. The mismatch repair (MMR) pathway is a DNA repair mechanism that recognizes and removes DNA replication errors. The loss of MMR proteins leads to an accumulation of DNA replication errors, which is termed microsatellite instability (MSI) (17). Proteins translated from genes with DNA replication errors can be immunogenic and provoke an antitumor immune response by increasing immune cell infiltration, which improves the sensitivity to PD-1/PD-L1 blockade therapy $(15,16)$.

The Cancer Genome Atlas Research Network revealed that PD-L1 gene amplification was higher in Epstein Barr virus $(\mathrm{EBV})^{+} \mathrm{GC}(18)$. In 1990, Burke et al (19) first reported an association between EBV status and the occurrence of GC with a characteristic lymphoepithelioma-like histology.

The expression of PD-L1 on the surface of TCs and in immune cells can be evaluated by immunohistochemistry (IHC) and serve as a predictive biomarker to identify patients that may benefit from immunotherapy; however, it has been identified that not all patients with a PD-L1 ${ }^{+}$status respond well to immunotherapy (20). Therefore, PD-L1 expression on TCs is currently considered an imperfect predictor of the response to immune checkpoint inhibitor therapy (15). For this reason, a number of studies have begun to investigate the tumor microenvironment, particularly focusing on the extent of tumor immune cell infiltration (7,15,21-23). An immunological classification of tumors into four different tumor microenvironment immune types (TMITs) based on PD-L1 status and low/high $\mathrm{CD}^{+}$tumor infiltrating lymphocyte (TIL) density has been proposed and validated in melanoma $(24,25)$. However, to the best of our knowledge, few studies have applied this classification in GC (26,27). To promote a more accurate selection of patients, the present study evaluated PD-L1 expression in the tumor microenvironment and quantified tumor infiltrating $\mathrm{CD}^{+} \mathrm{T}$ cell density in a number of GC cases characterized by MMR-D, HER2 and EBV status.

\section{Materials and methods}

Patients and tumor characteristics. A total of 46 males and 24 females (median age, 65.8 years; age range, 34-83 years) who underwent a curative gastrectomy for primary GC at the National Institute of Gastroenterology 'S. de Bellis' (Castellana Grotte, Italy) between 2014 and 2017 were included in the current study. The inclusion criteria included no previous chemotherapy, radiotherapy, Trastuzumab therapy or anti-PD-1/PD-L1 treatment prior to surgery. The pathological and clinical features of the patients are presented in Table I. The tumor site was proximal (cardias, corpus and fundus) in 37 patients and distal (antrum/pylorus) in 33 patients. According to the Lauren classification $(21,22,28)$, the histological types of the 70 GC cases included 36 diffuse and 34 intestinal. Other pathological characteristics of the GC cases are summarized in Table I. The present study was approved by the Review Board of National Institute of Gastroenterology (Castellana Grotte, Italy) and was conducted in accordance with the Declaration of Helsinki. Prior to enrollment, all participants provided written informed consent.

Paraffin-embedded sections (4- $\mu$ m-thick) were fixed with $10 \%$ neutral buffered formalin at room temperature for 24-48 h, immunostained, and in situ hybridization assays were performed. For each tumor, the histological subtype and tumor stage were reevaluated from hematoxylin and eosin (H\&E)-stained slides (29) using a light microscope (magnification, x5-40). Age, sex, nodal status and quantification of infiltrating immune cells (IICs), which encompass intratumoral and peritumoral lymphocytes, macrophages and plasma cells, were obtained from pathology reports and review of H\&E slides. IICs were evaluated as 'mild' when few cells (30-40 cells) were stained within the tumor and/or at the tumor-stroma interface (not deforming the distance between the glands), and 'marked' (>100 cells) when the infiltrate exhibited a greater density with a tendency to flow into plaques and infiltrate the neoplastic epithelium, deforming the distance between the glands.

Survival time was defined as the time from the date of surgery to the date of mortality or of the last successful interview. The median follow-up time was 24 months (range, 3-168 months). Patients who succumbed to the disease due to complications of the surgical procedure during the perioperative period or contact was lost prior to the first interview were excluded from the survival analysis.

IHC staining. IHC was performed on $10 \%$ neutral buffered formalin-fixed (room temperature for 24-48 h), paraffin-embedded sections (4- $\mu \mathrm{m}$-thick). IHC with anti-PD-L1 (cat. no. 13684S, clone E1L3N; Cell Signaling Technology, Inc., Danvers, MA, USA) diluted 1:800 at room temperature for $30 \mathrm{~min}$ was performed as previously described (23).

IHC with anti-CD8 (cat. no. M7103, Mab C8/144B; 1:200; Dako; Agilent Technologies, Inc., Santa Clara, CA, USA), anti-MutL homolog 1 (MLH1; cat. no. M3640, Mab ES05; 1:50; Dako Agilent Technologies, Inc. Santa Clara, CA, USA) and anti-HER2 (polyclonal antibody, cat. no. A0485; 1:200; Dako; Agilent Technologies, Inc.) was analyzed on an automated autostainer (Dako; Agilent Technologies, Inc.). Briefly, sections were dewaxed in an oven for $20 \mathrm{~min}$ at $60^{\circ} \mathrm{C}$, followed 
Table I. Association between PD-L1 expression and clinicopathological characteristics.

\begin{tabular}{|c|c|c|c|c|c|c|c|}
\hline \multirow[b]{2}{*}{ Characteristic } & \multirow[b]{2}{*}{$\begin{array}{c}\text { Total }(\mathrm{n}=70), \\
\mathrm{n}(\%)\end{array}$} & \multicolumn{3}{|c|}{ PD-L1 TCs } & \multicolumn{3}{|c|}{ PD-L1 IICs } \\
\hline & & $\begin{array}{c}\text { Positive }(\mathrm{n}=8), \\
\mathrm{n}(\%)\end{array}$ & $\begin{array}{c}\text { Negative }(\mathrm{n}=62), \\
\mathrm{n}(\%)\end{array}$ & P-value & $\begin{array}{c}\text { Positive }(\mathrm{n}=28) \\
\mathrm{n}(\%)\end{array}$ & $\begin{array}{c}\text { Negative }(\mathrm{n}=42), \\
\mathrm{n}(\%)\end{array}$ & P-value \\
\hline Sex & & & & $\mathrm{NS}^{\mathrm{b}}$ & & & $\mathrm{NS}^{\mathrm{b}}$ \\
\hline Female & $24(34.00)$ & $3(12.50)$ & $21(87.50)$ & & $9(37.50)$ & $15(62.50)$ & \\
\hline Male & $46(66.00)$ & $5(11.00)$ & $41(89.00)$ & & $19(41.00)$ & $27(59.00)$ & \\
\hline Age, years ${ }^{\mathrm{a}}$ & $65.83 \pm 10.63$ & $70.75 \pm 11.40$ & $65.19 \pm 10.45$ & $\mathrm{NS}^{\mathrm{d}}$ & $67.39 \pm 10.99$ & $65.12 \pm 10.27$ & $\mathrm{NS}^{\mathrm{d}}$ \\
\hline Tumor site & & & & $\mathrm{NS}^{\mathrm{b}}$ & & & $\mathrm{NS}^{\mathrm{b}}$ \\
\hline Distal & $33(47.00)$ & $3(9.00)$ & $30(91.00)$ & & $13(39.00)$ & $20(61.00)$ & \\
\hline Proximal & $37(53.00)$ & $5(13.50)$ & $32(86.50)$ & & $15(40.50)$ & $22(59.50)$ & \\
\hline Histological type & & & & $\mathrm{NS}^{\mathrm{b}}$ & & & $<0.05^{\mathrm{b}}$ \\
\hline Diffuse & $36(51.00)$ & $5(14.00)$ & $31(86.00)$ & & $9(25.00)$ & $27(75.00)$ & \\
\hline Intestinal & $34(49.00)$ & $3(9.00)$ & $31(91.00)$ & & $19(56.00)$ & $15(44.00)$ & \\
\hline Tumor grade (65) & & & & $\mathrm{NS}^{\mathrm{c}}$ & & & $\mathrm{NS}^{\mathrm{b}}$ \\
\hline $\mathrm{G} 1+\mathrm{G} 2$ & $12(17.00)$ & $0(0.00)$ & $12(100.00)$ & & $6(50.00)$ & $6(50.00)$ & \\
\hline G3 & $58(83.00)$ & $8(14.00)$ & $50(86.00)$ & & $22(38.00)$ & $36(62.00)$ & \\
\hline Pattern of growth & & & & $\mathrm{NS}^{\mathrm{b}}$ & & & $<0.05^{\mathrm{b}}$ \\
\hline Pushing & $14(20.00)$ & $3(21.00)$ & $11(79.00)$ & & $9(64.00)$ & $5(36.00)$ & \\
\hline Infiltrating & $56(80.00)$ & $5(9.00)$ & $51(91.00)$ & & $19(34.00)$ & $37(66.00)$ & \\
\hline Tumor Budding & & & & $\mathrm{NS}^{\mathrm{b}}$ & & & $\mathrm{NS}^{\mathrm{b}}$ \\
\hline Absent & $25(36.00)$ & $2(8.00)$ & $23(92.00)$ & & $13(52.00)$ & $12(48.00)$ & \\
\hline High & $45(64.00)$ & $6(13.00)$ & $39(87.00)$ & & $15(33.00)$ & $30(67.00)$ & \\
\hline IICs & & & & $<0.05^{\mathrm{b}}$ & & & $\mathrm{NS}^{\mathrm{b}}$ \\
\hline Mild & $45(64.00)$ & $2(4.00)$ & $43(96.00)$ & & $15(33.00)$ & $30(67.00)$ & \\
\hline Marked & $25(36.00)$ & $6(24.00)$ & $19(76.00)$ & & $13(52.00)$ & $12(48.00)$ & \\
\hline pT status & & & & $\mathrm{NS}^{\mathrm{c}}$ & & & $N S^{b}$ \\
\hline T1-T2 & $10(14.00)$ & $0(0.00)$ & $10(100.00)$ & & $4(40.00)$ & $6(60.00)$ & \\
\hline T3-T4 & $60(86.00)$ & $8(13.00)$ & $52(87.00)$ & & $24(40.00)$ & $36(60.00)$ & \\
\hline pN status & & & & $\mathrm{NS}^{\mathrm{b}}$ & & & $\mathrm{NS}^{\mathrm{b}}$ \\
\hline N0 & $13(19.00)$ & $3(23.00)$ & $10(77.00)$ & & $7(54.00)$ & $6(46.00)$ & \\
\hline $\mathrm{N}+$ & $57(81.00)$ & $5(9.00)$ & $52(91.00)$ & & $21(37.00)$ & $36(63.00)$ & \\
\hline MMR status & & & & $<0.05^{\mathrm{b}}$ & & & $<0.05^{\mathrm{c}}$ \\
\hline Deficient & $7(10.00)$ & $4(57.00)$ & $3(43.00)$ & & $7(100.00)$ & $0(0.00)$ & \\
\hline Proficient & $63(90.00)$ & $4(6.00)$ & $59(94.00)$ & & $21(33.00)$ & $42(67.00)$ & \\
\hline HER2 status & & & & $\mathrm{NS}^{\mathrm{b}}$ & & & $\mathrm{NS}^{\mathrm{b}}$ \\
\hline Positive & $19(27.00)$ & $2(10.50)$ & $17(89.50)$ & & $8(42.00)$ & $11(58.00)$ & \\
\hline Negative & $51(73.00)$ & $6(12.00)$ & $45(88.00)$ & & $20(39.00)$ & $31(61.00)$ & \\
\hline $\mathrm{CD}^{+}$ & & & & $<0.05^{\mathrm{b}}$ & & & $N S^{b}$ \\
\hline High & $16(23.00)$ & $5(31.00)$ & $11(69.00)$ & & $7(44.00)$ & $9(56.00)$ & \\
\hline Low & $54(77.00)$ & $3(5.50)$ & $51(94.50)$ & & $21(39.00)$ & $33(61.00)$ & \\
\hline PDL1 TCs & & & & - & & & $<0.05^{\mathrm{b}}$ \\
\hline Positive & $8(11.00)$ & - & - & & $6(75.00)$ & $2(25.00)$ & \\
\hline Negative & $62(89.00)$ & - & - & & $22(35.00)$ & $40(65.00)$ & \\
\hline
\end{tabular}

${ }^{a}$ Data presented as the mean \pm standard deviation. Data were analyzed by ${ }^{\mathrm{b}} \chi^{2}$ test, ${ }^{\mathrm{c}}$ Fisher's exact test or ${ }^{\mathrm{d}}$ Mann-Whitney U test. NS, not significant; PD-L1, programmed death-ligand 1; TC, tumor cell; IIC, infiltrating immune cell; HER2, human epidermal growth factor receptor 2; MMR, mismatch repair; CD8, cluster of differentiation 8.

by two washes with xylene. The sections were then rehydrated in a graded alcohol series (ethanol 99, 95, 70\%) and incubated with $3 \%$ hydrogen peroxide for $10 \mathrm{~min}$ at room temperature to block endogenous peroxidase activity. Sections were incubated 


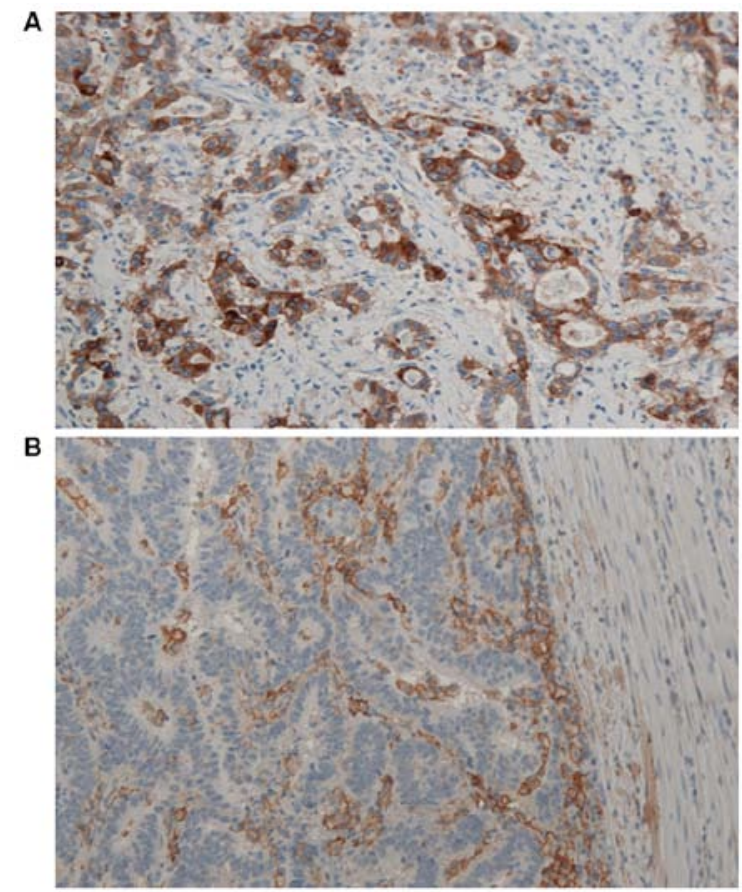

Figure 1. Programmed death-ligand 1 immunoreactivity on (A) tumor cells and (B) infiltrating immune cells. Magnification, x200.

with EDTA buffer $(\mathrm{pH} 8)$ at $98^{\circ} \mathrm{C}$ for $30 \mathrm{~min}$ for $\mathrm{CD} 8$ and MLH1 staining, and with $10 \mathrm{mM}$ citrate buffer $(\mathrm{pH} \mathrm{6})$ at $98^{\circ} \mathrm{C}$ for $30 \mathrm{~min}$ for HER2 staining. Monoclonal antibodies against CD8 and MLH1, and a polyclonal antibody against HER2 were incubated with the tissue sections (30 $\mathrm{min}$ at room temperature). PBS alone was used as the negative control. Dako Real ENVISION (cat. no. K5007; Dako; Agilent Technologies, Inc.) was used as visualization reagent (30 $\mathrm{min}$ at room temperature) and 3,3'-diaminobenzidine as chromogen (10 $\mathrm{min}$ at room temperature), according to the manufacturer's protocol.

PD-L1 immunoreactivity was randomly evaluated separately for TCs and IICs. Cells were counted in 5 randomly-selected fields. PD-L1 positivity was defined as $\geq 5 \%$ positive cells with membrane staining of any intensity (30). Cytoplasmic staining was not considered in the present study. For the evaluation of CD8, five fields (radius, $150 \mu \mathrm{m}$ ) in the intratumoral and peritumoral areas were selected. The number of positive cells was counted and the median number of $\mathrm{CD}^{+} \mathrm{T}$ lymphocytes was used as the cut-off value to classify low/high $\mathrm{CD}^{+}$TIL cases of GC (31). Tumors with no MLH1 staining in the TC nuclei were defined as MMR deficient, while tumors with nuclear staining were classified as MMR proficient (MMR-P). HER2 status was evaluated as previously described by Hofmann et al (32). All IHC staining was independently scored by two pathologists, Dr Raffaele Armentano and Dr Maria Lucia Caruso from the Department of Pathology (National Institute of Gastroenterology 'S. de Bellis', Research Hospital, Castellana Grotte, Italy).

HER2 chromogenic in situ hybridization (CISH). Cases with an IHC score of 2+ for HER2 were subjected to CISH for the evaluation of HER2 gene amplification. CISH was performed using the ZytoDot2C SPEC ERBB2/CEN17 probe kit (ZytoVision GmBH, Bremerhaven, Germany). Tissue sections (4 $\mu$ m-thick) were deparaffinized, incubated in pretreatment buffer for $15 \mathrm{~min}$ at $98-100^{\circ} \mathrm{C}$ and then washed with distilled water. Following enzymatic digestion for $15 \mathrm{~min}$ at room temperature, sections were dehydrated and incubated with $15 \mu \mathrm{l} \mathrm{HER} 2$ probe at $80^{\circ} \mathrm{C}$ for $5 \mathrm{~min}$ for denaturation and then at $37^{\circ} \mathrm{C}$ overnight for hybridization in a Thermobrite Hybridizer (StatSpin, Norwood, MA, USA). To prevent evaporation during incubation, standard coverslips and rubber cement were used. Following removal of the coverslips, the slides were washed in stringent wash buffer for $5 \mathrm{~min}$ at $75^{\circ} \mathrm{C}$. HER2 gene amplification was detected by sequential incubation with anti-DIG/DNP-mix, HRP/AP-Polymer-mix, AP-Red solution and HRP-Green solution. Finally, the slides were counterstained with hematoxylin (1 $\mathrm{min}$ at room temperature). Sections of breast cancer tissue known to be HER2-amplified were included as the positive control in each run.

The CISH signal was evaluated using a light microscope (magnification, $\mathrm{x} 40$ ) following review of the H\&E and IHC stained slides to identify areas of invasive $\mathrm{GC}$ with the strongest intensity of HER2 expression. The TC nuclei were evaluated to calculate the ratio of HER 2 to centromere 17 (CEN-17) signals. HER2 amplification was defined as a HER2/CEN-17 ratio $\geq 2$. If the ratio was $<2$, an average number of HER2 signals per cell count $<4$ indicated no HER2-amplification and an average number of signals per cell count $>6$ indicated HER 2 amplification. A mean average $\geq 4$ and $<6$ indicated an equivocal result. For an equivocal result performing IHC or CISH in a different patient sample was recommended (33). For the evaluation of HER2 status, cases with an IHC score of $3+$ or $2+$ with gene amplification were regarded as $\mathrm{HER} 2^{+}$and the remaining cases were defined as HER2-.

EBV status. CISH for EBV-encoded RNA (EBER) was performed using an RNAscope detection kit (Advanced Cell Diagnostics, Newark, CA, USA), according to the manufacturer's protocol. Tissue sections (4- $\mu$ m-thick) were baked at $60^{\circ} \mathrm{C}$ for $1 \mathrm{~h}$ and then deparaffinized. Deparaffinized slides were boiled for $10 \mathrm{~min}$ with citric buffer ( $\mathrm{pH}$ 6.0) for antigen retrieval. Slides were further permeabilized by protease treatment at $40^{\circ} \mathrm{C}$ for $30 \mathrm{~min}$ in a HybEZ hybridization oven (Advanced Cell Diagnostics), followed by hybridization with the target probe, amplification and detection by adding Amp1-4. Chromogenic detection was performed using 3,3'-diaminobenzidine followed by counterstaining with hematoxylin (1 min at room temperature). The kit included slides with HeLa cultured cells that were used as a positive or negative control depending on the probes adopted for hybridization. Samples stained brown in any TC nuclei were considered positive by using a light microscope (magnification, x10 and x20).

Statistical analysis. Continuous variables are presented as the mean \pm standard deviation and categorical variables are presented as the relative frequency (\%). To investigate associations between the categorical variables a $\chi^{2}$ test or Fisher's exact test were used, depending on the sample size. For non-parametric variables, analyses were performed by Mann-Whitney U test or Kruskal-Wallis test. The post hoc test used was Mann Whitney U with Bonferroni's correction test. For survival analysis, Kaplan-Meier analysis followed by a Wilcoxon-Breslow test and log-rank test was used to evaluate 
Table II. Association between EBV status, PD-L1 expression and CD8 ${ }^{+}$TILs density.

\begin{tabular}{|c|c|c|c|c|c|c|c|c|c|c|}
\hline \multirow[b]{2}{*}{ Characteristic } & \multirow[b]{2}{*}{$\begin{array}{c}\text { Total } \\
(\mathrm{n}=70), \\
\mathrm{n}(\%)\end{array}$} & \multicolumn{3}{|c|}{ PD-L1 TCs } & \multicolumn{3}{|c|}{ PD-L1 IICs } & \multicolumn{3}{|c|}{ CD8+ TILs } \\
\hline & & $\begin{array}{c}\text { Positive } \\
(\mathrm{n}=8) \\
\mathrm{n}(\%)\end{array}$ & $\begin{array}{c}\text { Negative } \\
(\mathrm{n}=62), \\
\mathrm{n}(\%)\end{array}$ & $P$-value ${ }^{a}$ & $\begin{array}{c}\text { Positive } \\
(\mathrm{n}=28) \\
\mathrm{n}(\%)\end{array}$ & $\begin{array}{c}\text { Negative } \\
(\mathrm{n}=42), \\
\mathrm{n}(\%)\end{array}$ & P-value ${ }^{b}$ & $\begin{array}{c}\text { High } \\
(\mathrm{n}=16), \\
\mathrm{n}(\%)\end{array}$ & $\begin{array}{c}\text { Low } \\
(\mathrm{n}=54), \\
\mathrm{n}(\%)\end{array}$ & P-value \\
\hline EBV status & & & & $<0.05$ & & & NS & & & $<0.05$ \\
\hline Positive & $2(3)$ & $2(100)$ & $0(0)$ & & $1(50)$ & $1(50)$ & & $2(100)$ & $0(0)$ & \\
\hline Negative & $68(97)$ & $6(0)$ & $62(100)$ & & $27(40)$ & $41(60)$ & & $14(21)$ & $54(79)$ & \\
\hline
\end{tabular}

Data were analyzed by ${ }^{a}$ Fisher's exact test or ${ }^{\mathrm{b}} \chi^{2}$ test. NS, not significant; PD-L1, programmed death-ligand 1; TC, tumor cell; IIC, infiltrating immune cell; EBV, Epstein Barr virus; TIL, tumor infiltrating lymphocyte.

the importance of single variables as prognostic factors associated with survival. $\mathrm{P}<0.05$ was considered to indicate a statistically significant difference. All statistical analysis was performed using STATA v.12.1 statistical software (StataCorp LP, College Station, TX, USA).

\section{Results}

PD-L1 immunohistochemical expression. Different percentages of PD-L1 were observed on TCs and IICs. All non-neoplastic gastric epithelia were identified to be PD-L1'. Among the 70 patients, 8 cases $(11 \%)$ were revealed to possess PD-L1 ${ }^{+}$TCs (Table I), the majority of which revealed a patchy pattern and rarely a diffused one. PD-L1 immunoreactivity on TCs was significantly associated with a greater density of IICs $(\mathrm{P}<0.05)$.

Furthermore, PD-L1 immunoreactivity was detected in IICs in $40 \%$ of GC cases (Table I) and was present within the tumor and at the interface between the tumor and surrounding stroma, with a prevalently patchy pattern. Representative images of PD-L1 immunohistochemical expression on TCs and IICs are presented in Fig. 1A and B, respectively. A total of six (75\%) TC PD-L1 ${ }^{+} \mathrm{GC}$ cases were also IIC PD-L1 ${ }^{+}$, while $35 \%$ of PD-L1 ${ }^{-}$GCs were IIC PD-L1 ${ }^{+}(\mathrm{P}<0.05$; Table I). PD-L1 positivity in IICs was significantly higher in the intestinal-type cases of GC compared with the diffuse-type cases (56 vs. $25 \%$, $\mathrm{P}<0.05$ ) and significantly higher in GC cases with a pushing pattern of growth compared with cases with an infiltrating pattern of growth (64 vs. $34 \%$; $\mathrm{P}<0.05$; Table I).

HER2 immunohistochemical expression and association with $P D$-L1 staining. HER 2 positivity was observed in 19 (27\%) samples (13 IHC score, 3+; 6 IHC score, 2+/gene amplified; Fig. 2A and B). A total of 51 HER2- tumors (25 IHC score, 0; 23 IHC score, 1; three IHC score, 2+ not amplified) were identified (Table II). Among the HER $2^{+}$samples, one (5\%) sample was PD-L1 $1^{+}$on TCs and IICs, seven (37\%) were positive only on IICs, and eleven (58\%) were negative on the two. No significant association was revealed between PD-L1 expression and HER2 status (Table I).

MLH1 immunohistochemical expression and association with PD-L1 staining. MLH1 deficiency was observed in

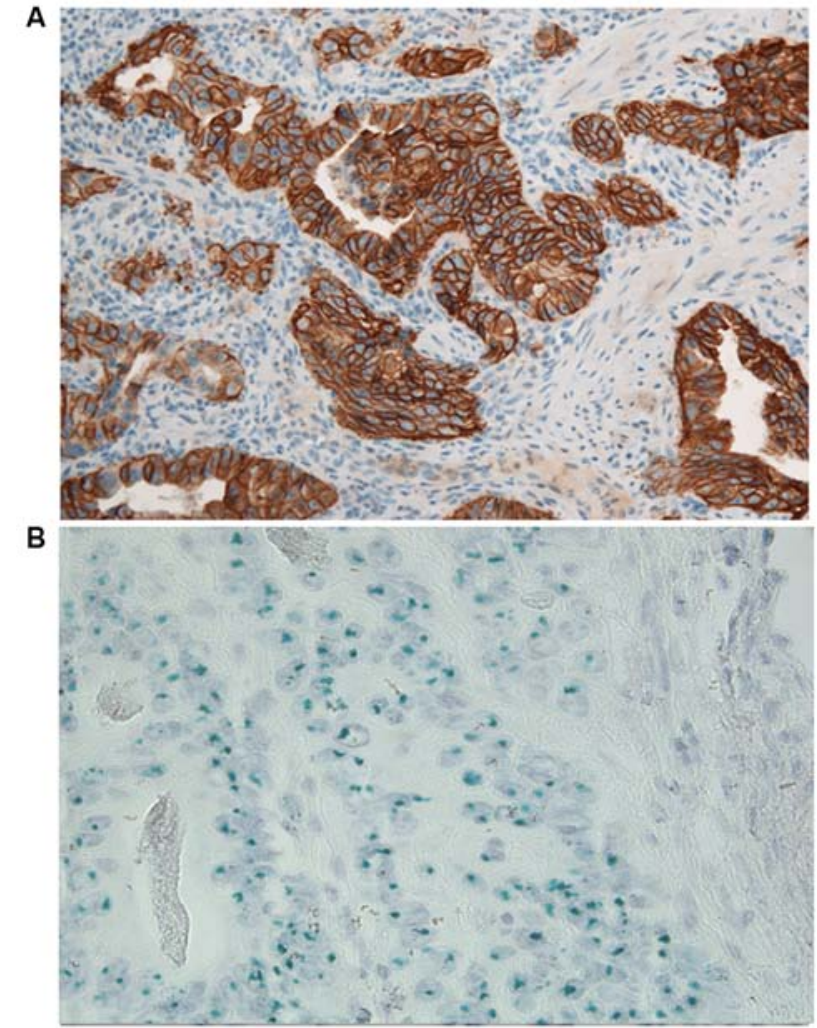

Figure 2. HER2 staining of gastric cancer cells. (A) HER2 immunohistochemical staining revealed a strong membranous pattern in neoplastic gastric cells with a score of 3+. Magnification, x200. (B) HER2 gene amplification is indicated by green clusters. Magnification, $x 400$. HER2, human epidermal growth factor receptor 2 .

seven $(10 \%)$ cases and was more frequent in PD-L1 ${ }^{+} \mathrm{GC}$ cases compared with PD-L1- GC cases. Four (57\%) GC cases that were PD-L1 ${ }^{+}$on TCs were MLH1 deficient, whereas $43 \%$ of PD-L1 ${ }^{-}$TC GC cases were MLH1 deficient $(\mathrm{P}<0.05$; Table I). All GC cases that were MMR deficient were PD- $\mathrm{L1}^{+}$on IICs, whereas $33 \%$ of MMR proficient GC cases were $\mathrm{PD}-\mathrm{L1}^{+}$on IICs $(\mathrm{P}<0.05$; Table I).

EBV status. Among the 70 patients, two (3\%) were positive for EBV infection. The histological features demonstrated poorly differentiated adenocarcinomas with a relatively rich 


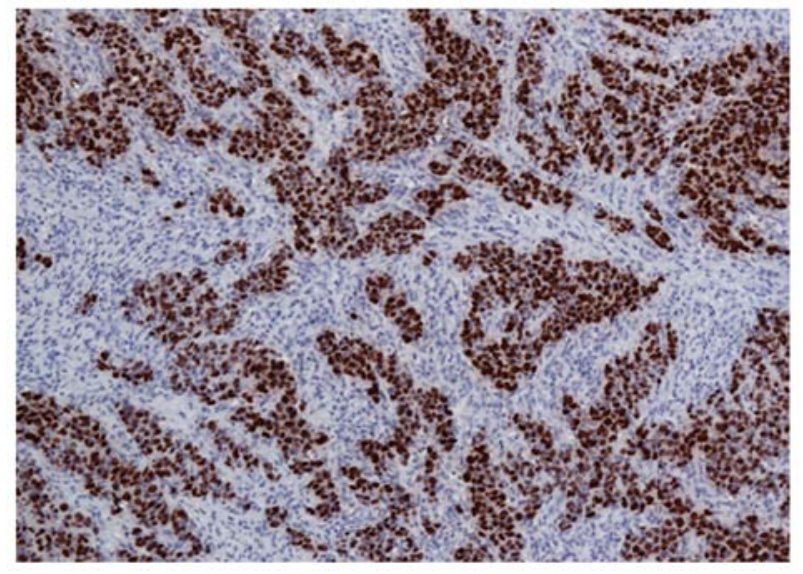

Figure 3. EBER CISH. EBER CISH demonstrated positive nuclei in the GC cells, which are surrounded by infiltrating lymphocytes. Magnification, x100. EBER, Epstein Barr virus-encoded RNA; CISH, chromogenic in situ hybridization.

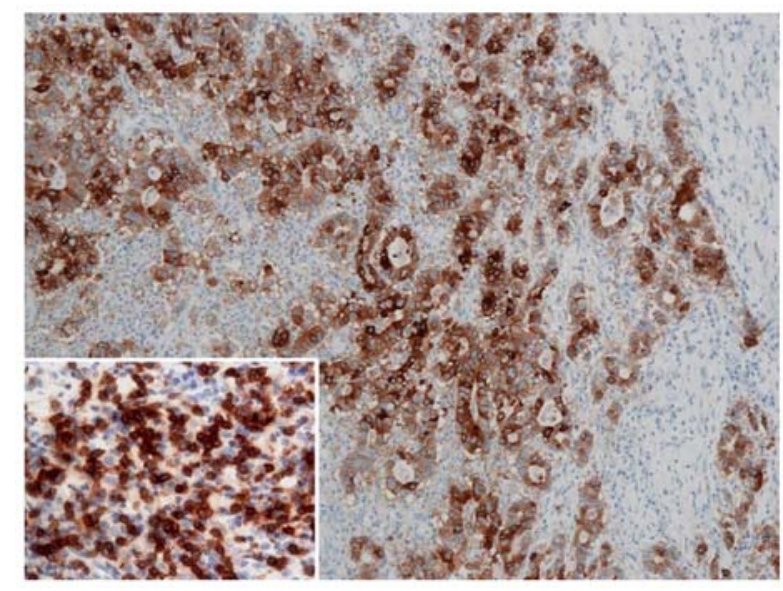

Figure 4. Staining of a tumor microenvironment immune type I GC sample. A programmed death-ligand $1^{+} \mathrm{GC}$ sample with a high cluster of differentiation $8^{+} \mathrm{T}$ lymphocyte density. Magnification, x100. Inset magnification, x200. $\mathrm{GC}$, gastric cancer.

lymphocyte infiltration. $\mathrm{EBV}^{+}$cases demonstrated intense nuclear staining. EBV positivity was observed in all tumoral cells aggregated in cords, which was clearly surrounded by EBV- lymphocytes (Fig. 3). No cases revealed positivity for $\mathrm{EBV}$ in the non-tumoral mucosa. The two $\mathrm{EBV}^{+}$samples were PD-L1 ${ }^{+}$on TCs $(\mathrm{P}<0.05$; Table II $)$.

CD8 immunohistochemical expression and TMITs. CD8 ${ }^{+}$ lymphocytes were present within tumor-cell nests and intratumoral and peritumoral stroma. In total, 16 (23\%) GC cases with a high $\mathrm{CD}^{+}$TILs density were identified and $54(77 \%)$ GC cases were revealed to have a low $\mathrm{CD} 8^{+}$TILs density. The cases that were PD- $\mathrm{L1}^{+}$on TCs more frequently featured a high $\mathrm{CD}^{+}$TILs density compared with the PD-L1' cases $\left(\mathrm{P}<0.05\right.$; Table I), and the two $\mathrm{EBV}^{+}$samples had a high $\mathrm{CD}^{+}$ high density $(\mathrm{P}<0.05$; Table II).

Based on the results of immunohistochemical expression of PD-L1 on TCs and CD8 ${ }^{+}$TILs density, the patients were categorized into the TMIT subgroups I-IV (34). The number and proportion in each TMIT were as follows: five (7\%) type I
(PD-L1 ${ }^{+} / \mathrm{CD}^{+}$high; Fig. 4), 51 (73\%) type II (PD-L1 $/ \mathrm{CD}^{+}$ low), three (4\%) type III (PD-L1 ${ }^{+} / \mathrm{CD}^{+}$low); eleven $(16 \%)$ type IV (PD-L1 $/ \mathrm{CD}^{+}$high). TMITs were significantly associated with pattern of growth, MMR status and EBV status (Table III).

Survival analysis. Survival data were available for 32 patients. The median follow-up time was 24 months (range, 3-168 months). During follow-up, 19 patients succumbed to GC and 13 were alive at the last date of follow-up. No significant associations were revealed with survival time (data not shown).

\section{Discussion}

Certain studies have demonstrated that PD-L1 is highly expressed on TCs and IICs in GC (26,35-38). However, the prognostic significance of PD-L1 remains controversial and its role as a therapeutic predictor requires further investigation.

A recent immune checkpoint blockade has received much attention for prolonging survival of patients with GC (39). In the KEYNOTE-059 study, a phase II trial, 259 patients with advanced refractory GC received Pembrolizumab until progression. The overall response rate was $15.5 \%$ in $\mathrm{PD}-\mathrm{L1}^{+}$ patients versus $5.5 \%$ in $\mathrm{PD}-\mathrm{L1}^{-}$patients. The threshold of PD-L1 immunohistochemical positivity was $1 \%$ and took into account staining on TCs and IICs (39). Following the results of this trial, the FDA approved Pembrolizumab for the treatment of patients with locally advanced or metastatic gastric, or gastroesophageal junction cancer in which TCs express PD-L1 (cut-off $>1 \%$ ) (6). Since not all patients who are determined as eligible for a targeted therapy demonstrate equal sensitivity, patient selection may be improved by taking into account PD-L1 expression in addition to other available biomarkers.

Furthermore, in accordance with the strategy of combining immunotherapy and targeted therapy, two recent multicenter studies have investigated the antitumor activity and safety of Pembrolizumab in combination with anti-HER2 agents in patients with HER2 ${ }^{+}$GC. The available data are at an early stage; however, high response rates and the preliminary overall survival data justify the interest associated with PD-1 inhibitors, which indicates immunotherapy may serve a significant role in GC (40).

To gain more in-depth information, the present study investigated PD-L1 expression in the tumor gastric microenvironment, and evaluated its association with HER2, MMR and EBV status. In agreement with previous studies $(21,35,36)$, the current study identified a higher expression level of PD-L1 on IICs $(40 \%)$ compared with TCs (11\%). In previous studies, PD-L1 on TCs was detected in $14-74 \%$ of patients with GCs $(22,26,34,38,41)$ compared with $30-88 \%$ on IICs $(21,35)$. The wide range of PD-L1 expression reported in previous studies may be explained by the different antibodies used to detect PD-L1 or the evaluation methods used to define PD-L1 positivity. The present study used the monoclonal antibody E1L3N from Cell Signaling Technology, Inc. as it is considered to have a higher sensitivity compared with other PD-L1 antibodies (42). The cut-off value for PD-L1 remains controversial. However, the majority of studies investigating GC evaluated the expression of PD-L1 using a 1 or $5 \%$ cut-off 
Table III. Association between TMIT and clinicopathological characteristics.

TMIT

\begin{tabular}{|c|c|c|c|c|c|c|}
\hline & & & & & & \\
\hline Characteristic & Total $(\mathrm{n}=70), \mathrm{n}(\%)$ & $\mathrm{I}(\mathrm{n}=5), \mathrm{n}(\%)$ & II $(\mathrm{n}=51), \mathrm{n}(\%)$ & III $(n=3), n(\%)$ & IV $(n=11), n(\%)$ & P-value \\
\hline Sex & & & & & & $N S^{b}$ \\
\hline Female & $24(34.00)$ & $1(4.00)$ & $15(62.50)$ & $2(8.00)$ & $6(25.50)$ & \\
\hline Male & $46(66.00)$ & $4(9.00)$ & $36(78.00)$ & $1(2.00)$ & $5(11.00)$ & \\
\hline Age, years ${ }^{a}$ & $65.83 \pm 10.63$ & $65.50 \pm 14.39$ & $64.60 \pm 10.66$ & $76.00 \pm 4.76$ & $67.67 \pm 9.53$ & $\mathrm{NS}^{\mathrm{c}}$ \\
\hline Tumor site & & & & & & $N S^{b}$ \\
\hline Distal & $33(47.00)$ & $1(3.00)$ & $24(73.00)$ & $2(6.00)$ & $6(82.00)$ & \\
\hline Proximal & $37(53.00)$ & $4(11.00)$ & $27(73.00)$ & $1(3.00)$ & $5(13.00)$ & \\
\hline Histological type & & & & & & $N S^{b}$ \\
\hline Diffuse & $36(51.00)$ & $4(11.00)$ & $25(69.00)$ & $1(3.00)$ & $6(17.00)$ & \\
\hline Intestinal & $34(49.00)$ & $1(3.00)$ & $26(76.00)$ & $2(6.00)$ & $5(15.00)$ & \\
\hline Tumor grade (65) & & & & & & $N S^{d}$ \\
\hline $\mathrm{G} 1+\mathrm{G} 2$ & $12(17.00)$ & $0(0.00)$ & $12(100.00)$ & $0(0.00)$ & $0(0.00)$ & \\
\hline $\mathrm{G} 3$ & $58(83.00)$ & $5(9.00)$ & $39(70.00)$ & $3(5.00)$ & $11(16.00)$ & \\
\hline Pattern of growth & & & & & & $<0.05^{\mathrm{d}}$ \\
\hline Pushing & $14(20.00)$ & $1(7.00)$ & $9(64.00)$ & $3(22.00)$ & $1(7.00)$ & \\
\hline Infiltrating & $56(80.00)$ & $4(7.00)$ & $42(75.00)$ & $0(0.00)$ & $10(18.00)$ & \\
\hline Tumor budding & & & & & & $\mathrm{NS}^{\mathrm{d}}$ \\
\hline Absent & $25(36.00)$ & $0(0.00)$ & $20(80.00)$ & $2(8.00)$ & $3(12.00)$ & \\
\hline High & $45(64.00)$ & $5(11.00)$ & $31(69.00)$ & $1(2.00)$ & $8(18.00)$ & \\
\hline pT status & & & & & & $\mathrm{NS}^{\mathrm{d}}$ \\
\hline $\mathrm{T} 1-\mathrm{T} 2$ & $10(14.00)$ & $0(0.00)$ & $9(90.00)$ & $0(0.00)$ & $1(10.00)$ & \\
\hline T3-T4 & $60(86.00)$ & $5(8.00)$ & $42(70.00)$ & $3(5.00)$ & $10(17.00)$ & \\
\hline pN status & & & & & & $\mathrm{NS}^{\mathrm{b}}$ \\
\hline No & $13(19.00)$ & $1(8.00)$ & $8(61.50)$ & $1(8.00)$ & $3(22.50)$ & \\
\hline $\mathrm{N}+$ & $57(81.00)$ & $4(7.00)$ & $43(75.00)$ & $2(3.50)$ & $8(14.50)$ & \\
\hline MMR status & & & & & & $<0.05^{\mathrm{d}}$ \\
\hline Deficient & $7(10.00)$ & $2(28.50)$ & $3(43.00)$ & $2(28.50)$ & $0(0.00)$ & \\
\hline Proficient & $63(90.00)$ & $3(5.00)$ & $48(76.00)$ & $1(2.00)$ & $11(17.00)$ & \\
\hline HER2 status & & & & & & $\mathrm{NS}^{\mathrm{b}}$ \\
\hline Positive & $19(27.00)$ & $1(5.00)$ & $15(79.00)$ & $1(5.00)$ & $2(10.50)$ & \\
\hline Negative & $51(73.00)$ & $4(8.00)$ & $36(71.00)$ & $2(4.00)$ & $9(17.00)$ & \\
\hline EBV status & & & & & & $<0.05^{\mathrm{d}}$ \\
\hline Positive & $2(3.00)$ & $2(100.00)$ & $0(0.00)$ & $0(0.00)$ & $0(0.00)$ & \\
\hline Negative & $68(97.00)$ & $3(4.00)$ & $51(75.00)$ & $3(4.00)$ & $11(17.00)$ & \\
\hline
\end{tabular}

${ }^{a}$ Data presented as the mean \pm standard deviation. Data were analyzed by ${ }^{\mathrm{b}} \chi^{2}$ test, ${ }^{\mathrm{C}}$ Kruskal-Wallis test or ${ }^{\mathrm{d}}$ Fisher's exact test. NS, not significant; TMIT, tumor microenvironment immune type; HER2, human epidermal growth factor receptor 2; MMR, mismatch repair; EBV, Epstein Barr virus.

value $(26,35,36,38)$. The latter value was used in the current study since minimal PD-L1 expression may have no effect on tumor biology (35).

Although the present study had a number of intrinsic limitations, our group has ample experience and has published various articles regarding different aspects of the PD-1/PD-L1 pathway $(23,43)$. A number of previous studies indicate that immunohistochemical positivity should be considered with caution $(32,33)$, particularly when it is used for the selection of patients suitable for therapy. Previously, extensive evaluations of markers with a cutoff $>1 \%$ have been replaced by different markers as they were ineffective, for example, the use of epidermal growth factor receptor and K-Ras has evolved over time in targeted therapy with Cetuximab $(44,45)$.

Furthermore, certain studies consider cytoplasmic positivity for the purpose of evaluating positive cases $(46,47)$. By contrast, it has repeatedly been stressed that membrane positivity is the only indicator of a receptor's presence (48). 
Unlike the strict immunohistochemical evaluation of HER2 in mammary and gastric carcinomas, to the best of our knowledge, precise guidelines do not exist for PD-L1, including for the evaluation of PD-L1 expression on biopsy material. However, the prevalent focal pattern of PD-L1 suggests immunohistochemical analysis should be performed on multiple biopsies to avoid underestimating the expression of PD-L1.

In the present study, the majority of patients who were PD-L1 ${ }^{+}$on TC demonstrated PD-L1 positivity on IICs, which suggests that $\mathrm{PD}-\mathrm{L} 1$ expression in GCs is predominantly controlled by an adaptive immune resistance induced by immune cells via interferon- $\gamma$ secretion rather than by an intrinsic pathway (25). However, $31 \%$ of GCs were only PD-L1+ on IICs. PD-L1 is constitutively expressed in certain immune cells, including lymphocytes, macrophages and dendritic cells (49); however, it can also be induced by inflammatory cytokines (25). This likely reflects the combined effect of innate and adaptive, and cellular and soluble factors present in the tumor microenvironment (50). Certain studies have reported a positive association between PD-L1 expression on IICs and the response to PD-1 inhibitor treatment, which supports the use of PD-L1 expression as a predictive marker for immunotherapy $(42,51)$.

The positive association between PD-L1 expression on IICs and intestinal type GC may be due to the different pathogenesis of intestinal and diffuse histological types (52). The present study suggests that the multistep carcinogenic pathway, caused by carcinogens, from chronic gastric inflammation to intestinal type cancer could exert a greater and more prolonged stimulation of the immune system, including mucosa-associated lymphoid tissue, which eventually becomes self-limited and induces the expression of PD-L1.

In agreement with Kawazoe et al (47), the present study demonstrated that PD-L1 expression on TCs and/or IICs was not associated with clinicopathological characteristics of poor prognosis. In addition, PD-L1 expression on TCs was identified to be significantly associated with high CD8 ${ }^{+}$TIL density, which is associated with adaptive resistance. This indicates that the tumor cells expressing PDL-1 in response to the cytokines produced by the TILs are able to escape the immune response of the host through the binding to PD-1 in T cells (25). By contrast, no association was revealed between PD-L1 expression on IICs and high CD8 ${ }^{+}$TILs, which indicates that the IICs are not only composed of $\mathrm{CD}^{+} \mathrm{T}$ lymphocytes.

Furthermore, the current study evaluated the MMR status only by an assessment of MLH1 protein expression, as it was lost in $>90 \%$ of GCs with MSI (4). In the present study, the percentage of GCs with MMR-D (10\%) was consistent with a previous study that reported a frequency of $10-20 \%$ (4). In agreement with previous studies $(35,43)$, the current study demonstrated a significant association between MMR-D and PD-L1 expression on TCs and IICs. This finding can be explained as MMR deficient tumors are considered to be highly immunogenic due to the heavy mutation-associated-neoantigens burden, which is responsible for adaptive immune resistance $(15,16)$.

A number of studies support the hypothesis that TILs exhibit a tumor prognostic value and an 'immunoscore', which takes into account $\mathrm{CD}^{+}$and $\mathrm{CD}^{+}$TILs, has been demonstrated to be a powerful prognostic and therapeutic indicator $(21,53)$. To identify which patients are suitable for immunotherapy, a new classification of tumors has recently been proposed, based on PD-L1 status on TCs and the presence/absence of TILs $(24,25)$. This classification was developed to improve understanding of the tumor immune microenvironment. However, the tumor microenvironment is particularly complex and several critical issues have to be considered for the interaction between PD-L1 and TILs, including density, location and lymphocytes subpopulations. This classification was considered too simplistic and was later modified using immunohistochemical positivity for CD8 as a surrogate marker for TILs (34). The new classification consists of the four following TMITs: I (PD- $1^{+} / \mathrm{CD} 8^{+}$high, adaptive immune resistance), II (PD-L1 $/ \mathrm{CD}^{+}$low, immune ignorance type), III (PD- $\mathrm{L1}^{+} / \mathrm{CD}^{+}$low, intrinsic induction of PD-L1) and IV (PD-L1 $/ \mathrm{CD} 8^{+}$high, tolerant tumors), which indicates the role of other suppressors in promoting immune tolerance (24). The proportion of various cancer types that fit into each of these types likely depends on other genetic aberrations and oncogene drivers, as well as the type of tissue in which they originate (25).

Malignant melanoma has been extensively studied and a high proportion of type I and II microenvironments has been reported (25). Clinical studies have demonstrated that $\sim 38 \%$ of patients with advanced melanoma with a type I profile are sensitive to anti-PD-L1 treatment. By contrast, patients with melanoma with a type II tumor microenvironment are not responsive to checkpoint blockade (25). To the best of our knowledge, similar information regarding GC remains unknown.

The present study classified patients with GC into the TMITs I-IV based on the results of PD-L1 expression on TCs and CD8 ${ }^{+}$TIL density. In agreement with previous studies $(21,54)$, it was identified that the majority of GC cases belong to TMIT II (73\%), which is the immune ignorance type. Previous studies that have described TMITs in GC revealed that the highest percentage of tumors were type II $(21,27)$; however, a higher frequency was identified in the present study. This may be due to the cut-off value of $5 \%$ used in the current study, which can lead to the identification of a larger number of negative cases compared with studies that used a cut-off value of $1 \%$. A low level of CD8 $8^{+}$TILs may partially explain the high mortality rate of patients with GC and restrict the use of antibodies that target immune checkpoints.

In agreement with previous studies $(21,27)$, the current study demonstrated that TMIT I GC cases are associated with EBV infection and MSI status, which are characterized by heavy lymphocytic infiltration (19). EBERs are the most abundant latency-associated transcripts in EBV-infected cells (55). Notably, EBERs are very stable in formalin-fixed paraffin-embedded tissues, which makes them sensitive EBV markers (55). CISH of EBER has been reported to be beneficial for the detection of EBV-infected cells (56). The RNAscope is a novel RNA-ISH platform that makes use of a novel probe design strategy and a hybridization-based signal amplification system that can theoretically yield up to 8,000 labels for each target RNA molecule, resulting in high definition signals (57). The percentage of positive EBER GC cases identified in the present study (3\%) was lower compared with that reported in a previous study (7-10\%) (18), which may be due to the limited 
number of lymphoepithelioma-like cases. In the current study, EBER was exclusively detected in the TC compartment, and not in lymphocytes and stromal cells, which is consistent with a previous study (18). Furthermore, a high rate of infiltration of $\mathrm{CD}^{+} \mathrm{T}$ cells is a characteristic of $\mathrm{EBV}^{+} \mathrm{GC}$ cases (58). The $\mathrm{EBV}^{+} \mathrm{GC}$ cases in the present study were associated with high densities of CD8 ${ }^{+}$TILs and belonged to TMIT I. TMIT I status involves adaptive immune escape responses and previous studies suggest that GC cases with this signature can be reversed by immune checkpoint blockade $(24,59)$.

TMIT III was identified as the least frequent category, which demonstrates that constitutive expression of PD-L1 on TCs through oncogenic signaling is not an important phenomenon in GC. This group could include patients who exhibit positive PD-L1 expression on neoplastic cells but do not respond to therapy (25). GC cases of TMIT IV contain a high CD8 ${ }^{+}$TILs density, however, do not demonstrate obvious adaptive resistance, as neoplastic gastric cells do not express PD-L1.

Tumeh et al (60) reported that for patients with stage III malignant melanoma, a predictive marker of clinical response to PD-1 blockade is the density of CD8 ${ }^{+}$TILs and not PD-L1 expression itself. This could explain the responsiveness to therapy of patients with negative PD-L1 expression on TCs.

Furthermore, in the present study, an association between PD-L1 and HER2 was not identified. Li et al (22) reported that PD-L1 expression on GC TCs is associated with HER2 expression; however, the study did not specify if the samples were HER2- or HER2 ${ }^{+}$. Oki et al (61) demonstrated that PD-L1+ GC cases were associated with an increasing HER2 score. These previous studies did not make a distinction between HER $2^{+}$versus HER2- samples. Despite correctly evaluating the positivity of HER2, according to Hofmann's criteria (32), Böger et al (35), Ju et al (30) and Wang et al (36) reported contradictory results regarding the association between PD-L1 and HER2.

In agreement with a previous study (62), the present study revealed that $10.5 \%$ of $\mathrm{HER}^{+} \mathrm{GC}$ samples versus

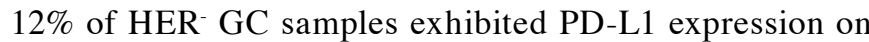
TCs. No HER $2^{+}$sample was identified to be MMR deficient. The Research Network of The Cancer Genome Atlas data demonstrated that HER2 amplification was more common in tumors with chromosomal instability compared with microsatellite instability (18). Therefore, the positive association between PD-L1 and MMR status may explain the low probability of identifying GC samples positive for PD-L1 and HER2.

In GC, the association between PD-L1 expression and survival remains to be fully understood $(35,41,63,64)$. Fang et al (41) demonstrated that PD-L1 expression on TCs was not associated with overall survival, while patients with PD-L1 expression on TILs had a significantly shorter 5-year overall survival rate compared those with negative PD-L1 expression. A recent study of a large cohort of Caucasian patients with GC revealed that PD-L1 expression in tumor and stromal immune cells was associated with improved survival (34), while previous studies of an Asian population revealed a poor prognostic role of PD-L1 $(63,64)$. Apart from the difference of ethnicity, these studies were performed in heterogeneous populations, which contained patients with various stages of cancer that were undergoing different treatment strategies in different clinical settings.

In the present study, the small number of samples did not allow any significant differences in survival time to be identified between PD-L1 ${ }^{+}$and PDL1 ${ }^{-}$patients, even when taking into account the TMITs. In conclusion, the current results, which require validation with a larger sample size, may highlight a subset of patients with GC who exhibit PD-L1 expression on TCs, a high density of CD8 ${ }^{+}$TILs and MMR deficiency. To the best of our knowledge, for the first time the present study demonstrated that a positivity of EBER, detected using the RNAscope method, may be useful for predicting the effectiveness of anti-PD-1/PD-L1 antibody therapy.

\section{Acknowledgements}

Not applicable

\section{Funding}

This study was supported by the Italian Ministry of Health, Ricerca Corrente 2018.

\section{Availability of data and materials}

All data analyzed during this study are included in this article.

\section{Authors' contributions}

AMV and MLC conceived and designed the study. FDP and SC collected samples for immunohistochemical analysis and performed the immunohistochemical staining. AMV and RA were responsible for immunohistochemical evaluation. VG analyzed the data. FDP prepared the figures. AMV and MLC wrote the paper. All authors read and approved the final manuscript.

\section{Ethics approval and consent to participate}

The present study was approved by the Institutional Review Board (National Institute of Gastroenterology 'S. de Bellis', Research Hospital, Castellana Grotte, Bari, Italy) and was conducted in accordance with the Declaration of Helsinki. Prior to enrollment, all parti cipants provided written informed consent.

\section{Patient consent for publication}

Not applicable.

\section{Competing interests}

The authors declare that they have no competing interests.

\section{References}

1. Ferlay J, Soerjomataram I, Dikshit R, Eser S, Mathers C, Rebelo M, Parkin DM, Forman D and Bray F: Cancer incidence and mortality worldwide: Sources, methods and major patterns in GLOBOCAN 2012. Int J Cancer 136: E359-E386, 2015. 
2. Yazici O, Sendur MA, Ozdemir N and Aksoy S: Targeted therapies in gastric cancer and future perspectives. World $\mathrm{J}$ Gastroenterol 22: 471-489, 2016.

3. Wong SS, Kim KM, Ting JC, Yu K, Fu J, Liu S, Cristescu R, Nebozhyn M, Gong L, Yue YG, et al: Genomic landscape and genetic heterogeneity in gastric adenocarcinoma revealed by whole-genome sequencing. Nat Commun 5: 5477, 2014.

4. Lee J and Kim KM: Biomarkers for gastric cancer: Molecular classification revisited. Precis Future Med 1: 59-68, 2017.

5. Setia N, Agoston AT, Han HS, Mullen JT, Duda DG, Clark JW, Deshpande V, Mino-Kenudson M, Srivastava A, Lennerz JK, et al: A protein and mRNA expression-based classification of gastric cancer. Mod Pathol 29: 772-784, 2016.

6. Fashoyin-Aje L, Donoghue M, Chen H, He K, Veeraraghavan J, Goldberg KB, Keegan P, McKee AE and Pazdur R: FDA Approval Summary: Pembrolizumab for Recurrent Locally Advanced or Metastatic Gastric or Gastroesophageal Junction Adenocarcinoma Expressing PD-L1. Oncologist 24: 103-109, 2019.

7. Kulangara K, Zhang N, Corigliano E, Guerrero L, Waldroup S, Jaiswal D, Ms MJ, Shah S, Hanks D, Wang J, et al: Clinical Utility of the Combined Positive Score for Programmed Death Ligand-1 Expression and the Approval of Pembrolizumab for Treatment of Gastric Cancer. Arch Pathol Lab Med 143: 330-337, 2019.

8. Topalian SL, Drake CG and Pardoll DM: Immune checkpoint blockade: A common denominator approach to cancer therapy. Cancer Cell 27: 450-461, 2015.

9. Nishimura H and Honjo T: PD-1: An inhibitory immunoreceptor involved in peripheral tolerance. Trends Immunol 22: 265-268, 2001.

10. Boussiotis VA: Molecular and biochemical aspects of the PD-1 checkpoint pathway. N Engl J Med 375: 1767-1778, 2016.

11. Fanoni D, Tavecchio S, Recalcati S, Balice Y, Venegoni L, Fiorani R, Crosti C and Berti E: New monoclonal antibodies against B-cell antigens: Possible new strategies for diagnosis of primary cutaneous B-cell lymphomas. Immunol Lett 134: 157-160, 2011

12. Terme M, Ullrich E, Aymeric L, Meinhardt K, Desbois M, Delahaye N, Viaud S, Ryffel B, Yagita H, Kaplanski G, et al: IL-18 induces PD-1-dependent immunosuppression in cancer. Cancer Res 71: 5393-5399, 2011.

13. Hino R, Kabashima K, Kato Y, Yagi H, Nakamura M, Honjo T, Okazaki T and Tokura Y: Tumor cell expression of programmed cell death-1 ligand 1 is a prognostic factor for malignant melanoma. Cancer 116: 1757-1766, 2010.

14. Sui H, Ma N, Wang Y, Li H, Liu X, Su Y and Yang J: Anti-PD-1/PD-L1 Therapy for Non-Small-Cell Lung Cancer: Toward Personalized Medicine and Combination Strategies. J Immunol Res 2018: 6984948, 2018.

15. Llosa NJ, Cruise M, Tam A, Wicks EC, Hechenbleikner EM, Taube JM, Blosser RL, Fan H, Wang H, Luber BS, et al: The vigorous immune microenvironment of microsatellite instable colon cancer is balanced by multiple counter-inhibitory checkpoints. Cancer Discov 5: 43-51, 2015.

16. Xiao Y and Freeman GJ: The microsatellite instable subset of colorectal cancer is a particularly good candidate for checkpoint blockade immunotherapy. Cancer Discov 5: 16-18, 2015.

17. Caruso ML: Role of mismatch repair proteins and microsatellite instability in colon carcinoma. In: Immunohistochemistry and in situ hybridization of human carcinomas. Hayat MA (ed) Elsevier Academic Press, New York, NY, pp215-226, 2005

18. Bass AJ, Thorsson V, Shmulevich I, Reynolds SM, Miller M, Bernard B, Hinoue T, Laird PW, Curtis C, Shen H, et al; Cancer Genome Atlas Research Network: Comprehensive molecular characterization of gastric adenocarcinoma. Nature 513: 202-209, 2014.

19. Burke AP, Yen TS, Shekitka KM and Sobin LH: Lymphoepithelial carcinoma of the stomach with Epstein-Barr virus demonstrated by polymerase chain reaction. Mod Pathol 3: 377-380, 1990.

20. Tie Y, Ma X, Zhu C, Mao Y, Shen K, Wei X, Chen Y and Zheng H: Safety and efficacy of nivolumab in the treatment of cancers: A meta-analysis of 27 prospective clinical trials. Int J Cancer 140: 948-958, 2017.

21. Kim KJ, Yang HK, Kim WH and Kang GH: Combined prognostic effect of PD-L1 expression and immunoscore in microsatellite-unstable advanced gastric cancers. Oncotarget 8 : 58887-58902, 2017.

22. Li Z, Lai Y, Sun L, Zhang X, Liu R, Feng G, Zhou L, Jia L, Huang X, Kang Q, et al: PD-L1 expression is associated with massive lymphocyte infiltration and histology in gastric cancer. Hum Pathol 55: 182-189, 2016.
23. Valentini AM, Di Pinto F, Cariola F, Guerra V, Giannelli G, Caruso ML and Pirrelli M: PD-L1 expression in colorectal cancer defines three subsets of tumor immune microenvironments. Oncotarget 9: 8584-8596, 2018.

24. Taube JM1, Anders RA, Young GD, Xu H, Sharma R, McMiller TL, Chen S, Klein AP, Pardoll DM, Topalian SL and Chen L: Colocalization of inflammatory response with B7-h1 expression in human melanocytic lesions supports an adaptive resistance mechanism of immune escape. Sci Transl Med 4: 127ra37, 2012.

25. Teng MWL, Ngiow SF, Ribas A and Smyth MJ: Classifying Cancers Based on T-cell Infiltration and PD-L1. Cancer Res 75: 2139-2145, 2015 .

26. Koh J, Ock CY, Kim JW, Nam SK, Kwak Y, Yun S, Ahn SH, Park DJ, Kim HH, Kim WH, et al: Clinicopathologic implications of immune classification by PD-L1 expression and CD8-positive tumor-infiltrating lymphocytes in stage II and III gastric cancer patients. Oncotarget 8: 26356-26367, 2017.

27. Ma J, Li J, Hao Y, Nie Y, Li Z, Qian M, Liang Q, Yu J, Zeng M and $\mathrm{Wu} \mathrm{K}$ : Differentiated tumor immune microenvironment of Epstein-Barr virus-associated and negative gastric cancer: Implication in prognosis and immunotherapy. Oncotarget 8: 67094-67103, 2017.

28. Lauren P: The two histological main types of gastric carcinoma: Diffuse and so-called intestinal-type carcinoma. An attempt at a histo-clinical classification. Acta Pathol Microbiol Scand 64: 31-49, 1965.

29. Feldman AT and Wolfe D: Tissue processing and hematoxylin and eosin staining. Methods Mol Biol 1180: 31-43, 2014.

30. Ju X, Shen R, Huang P, Zhai J, Qian X, Wang Q and Chen M: Predictive relevance of PD-L1 expression with pre-existing TILs in gastric cancer. Oncotarget 8: 99372-99381, 2017.

31. Wang W, Wang K, Chen Z, Chen L, Guo W, Liao P, Rotroff D, Knepper TC, Liu Z, Zhang W, et al: Immunoclassification characterized by CD8 and PD-L1 expression is associated with the clinical outcome of gastric cancer patients. Oncotarget 9: 12164-12173, 2018

32. Hofmann M, Stoss O, Shi D, Büttner R, van de Vijver M, Kim W, Ochiai A, Rüschoff $\mathbf{J}$ and Henkel T: Assessment of a HER2 scoring system for gastric cancer: Results from a validation study. Histopathology 52: 797-805, 2008.

33. Bartley AN, Washington MK, Colasacco C, Ventura CB, Ismaila N, Benson AB III, Carrato A, Gulley ML, Jain D, Kakar S, et al: HER2 Testing and Clinical Decision Making in Gastroesophageal Adenocarcinoma: Guideline From the College of American Pathologists, American Society for Clinical Pathology, and the American Society of Clinical Oncology. J Clin Oncol 35: 446-464, 2017.

34. Ock CY, Keam B, Kim S, Lee JS, Kim M, Kim TM, Jeon YK, Kim DW, Chung DH and Heo DS: Pan-Cancer Immunogenomic Perspective on the Tumor Microenvironment Based on PD-L1 and CD8 T-Cell Infiltration. Clin Cancer Res 22: 2261-2270, 2016

35. Böger C, Behrens HM, Mathiak M, Krüger S, Kalthoff $H$ and Röcken C: PD-L1 is an independent prognostic predictor in gastric cancer of Western patients. Oncotarget 7: 24269-24283, 2016.

36. Wang L, Zhang Q, Ni S, Tan C, Cai X, Huang D and Sheng W: Programmed death-ligand 1 expression in gastric cancer: Correlation with mismatch repair deficiency and HER2-negative status. Cancer Med 7: 2612-2620, 2018.

37. Wu Y, Cao D, Qu L, Cao X, Jia Z, Zhao T, Wang Q and Jiang J: PD-1 and PD-L1 co-expression predicts favorable prognosis in gastric cancer. Oncotarget 8: 64066-64082, 2017.

38. Harada K, Dong X, Estrella JS, Correa AM, Xu Y, Hofstetter WL, Sudo K, Onodera H, Suzuki K, Suzuki A, et al: Tumor-associated macrophage infiltration is highly associated with PD-L1 expression in gastric adenocarcinoma. Gastric Cancer 21: 31-40, 2018.

39. Fuchs CS, Doi T, Jang RW, Muro K, Satoh T, Machado M, Sun W, Jalal SI, Shah MA, Metges JP, et al: Safety and Efficacy of Pembrolizumab Monotherapy in Patients With Previously Treated Advanced Gastric and Gastroesophageal Junction Cancer: Phase 2 Clinical KEYNOTE-059 Trial. JAMA Oncol 4: e180013, 2018.

40. Apicella M, Corso S and Giordano S: Targeted therapies for gastric cancer: Failures and hopes from clinical trials. Oncotarget 8: 57654-57669, 2017.

41. Fang W, Chen Y, Sheng J, Zhou T, Zhang Y, Zhan J, Liu L, Huang J, Peng P and Zhang L: Association between PD-L1 Expression on Tumour-Infiltrating Lymphocytes and Overall Survival in Patients with Gastric Cancer. J Cancer 8: 1579-1585, 2017. 
42. Ying L, Yan F, Meng Q, Yuan X, Yu L, Williams BRG, Chan DW, Shi L, Tu Y, Ni P, et al: Understanding immune phenotypes in human gastric disease tissues by multiplexed immunohistochemistry. J Transl Med 15: 206, 2017.

43. Cavalcanti E, Armentano R, Valentini AM, Chieppa M and Caruso ML: Role of PD-L1 expression as a biomarker for GEP neuroendocrine neoplasm grading. Cell Death Dis 8: e3004, 2017.

44. Saltz LB, Meropol NJ, Loehrer PJ Sr, Needle MN, Kopit J and Mayer RJ: Phase II trial of cetuximab in patients with refractory colorectal cancer that expresses the epidermal growth factor receptor. J Clin Oncol 22: 1201-1208, 2004.

45. Cunningham D, Humblet Y, Siena S, Khayat D, Bleiberg H, Santoro A, Bets D, Mueser M, Harstrick A, Verslype C, et al: Cetuximab monotherapy and cetuximab plus irinotecan in irinotecan-refractory metastatic colorectal cancer. N Engl J Med 351: 337-345, 2004.

46. Gu L, Chen M, Guo D, Zhu H, Zhang W, Pan J, Zhong X, Li X, Qian H and Wang X: PD-L1 and gastric cancer prognosis: A systematic review and meta-analysis. PLoS One 12: e0182692, 2017.

47. Kawazoe A, Kuwata T, Kuboki Y, Shitara K, Nagatsuma AK, Aizawa M, Yoshino T, Doi T, Ohtsu A and Ochiai A: Clinicopathological features of programmed death ligand 1 expression with tumor-infiltrating lymphocyte, mismatch repair, and Epstein-Barr virus status in a large cohort of gastric cancer patients. Gastric Cancer 20: 407-415, 2017.

48. Udall M, Rizzo M, Kenny J, Doherty J, Dahm S, Robbins P and Faulkner E: PD-L1 diagnostic tests: A systematic literature review of scoring algorithms and test-validation metrics. Diagn Pathol 13: 12, 2018

49. Kwon D, Kim S, Kim PJ, Go H, Nam SJ, Paik JH, Kim YA, Kim TM, Heo DS, Kim CW, et al: Clinicopathological analysis of programmed cell death 1 and programmed cell death ligand 1 expression in the tumour microenvironments of diffuse large B cell lymphomas. Histopathology 68: 1079-1089, 2016.

50. Scognamiglio G, De Chiara A, Di Bonito M, Tatangelo F, Losito NS, Anniciello A, De Cecio R, D'Alterio C, Scala S, Cantile M, et al: Variability in Immunohistochemical Detection of Programmed Death Ligand 1 (PD-L1) in Cancer Tissue Types. Int J Mol Sci 17: E790, 2016.

51. Romano $\mathrm{E}$ and Romero P: The therapeutic promise of disrupting the PD-1/PD-L1 immune checkpoint in cancer: Unleashing the CD8 T cell mediated anti-tumor activity results in significant, unprecedented clinical efficacy in various solid tumors. J Immunother Cancer 3: 15, 2015.

52. Grabsch HI and Tan P: Gastric cancer pathology and underlying molecular mechanisms. Dig Surg 30: 150-158, 2013.

53. Jiang Y, Zhang Q, Hu Y, Li T, Yu J, Zhao L, Ye G, Deng H, Mou T, Cai S, et al: ImmunoScore Signature: A Prognostic and Predictive Tool in Gastric Cancer. Ann Surg 267: 504-513, 2018.
54. Choi YY, Bae JM, An JY, Kwon IG, Cho I, Shin HB, Eiji T, Aburahmah M, Kim HI, Cheong JH, et al: Is microsatellite instability a prognostic marker in gastric cancer? A systematic review with meta-analysis. J Surg Oncol 110: 129-135, 2014.

55. Shinozaki-Ushiku A, Kunita A and Fukayama M: Update on Epstein-Barr virus and gastric cancer (review). Int J Oncol 46: 1421-1434, 2015. (review)

56. Niedobitek G, Young LS, Sam CK, Brooks L, Prasad U and Rickinson AB: Expression of Epstein-Barr virus genes and of lymphocyte activation molecules in undifferentiated nasopharyngeal carcinomas. Am J Pathol 140: 879-887, 1992.

57. Wang F, Flanagan J, Su N, Wang LC, Bui S, Nielson A, Wu X, Vo HT, Ma XJ and Luo Y: RNAscope: A novel in situ RNA analysis platform for formalin-fixed, paraffin-embedded tissues. J Mol Diagn 14: 22-29, 2012

58. Nishikawa J, Iizasa H, Yoshiyama H, Shimokuri K, Kobayashi Y, Sasaki S, Nakamura M, Yanai H, Sakai K, Suehiro Y, et al: Clinical Importance of Epstein-Barr Virus-Associated Gastric Cancer. Cancers (Basel) 10: pii: E167, 2018.

59. Taube JM, Klein A, Brahmer JR, Xu H, Pan X, Kim JH, Chen L, Pardoll DM, Topalian SL and Anders RA: Association of PD-1, PD-1 ligands, and other features of the tumor immune microenvironment with response to anti-PD-1 therapy. Clin Cancer Res 20: 5064-5074, 2014

60. Tumeh PC, Harview CL, Yearley JH, Shintaku IP, Taylor EJ, Robert L, Chmielowski B, Spasic M, Henry G, Ciobanu V, et al: PD-1 blockade induces responses by inhibiting adaptive immune resistance. Nature 515: 568-571, 2014

61. Oki E, Okano S, Saeki H, Umemoto Y, Teraishi K, Nakaji Y, Ando K, Zaitsu Y, Yamashita N, Sugiyama M, et al: Protein Expression of Programmed Death 1 Ligand 1 and HER2 in Gastric Carcinoma. Oncology 93: 387-394, 2017.

62. Gonzalez RS, Messing S, Tu X, McMahon LA and Whitney-Miller CL: Immunohistochemistry as a surrogate for molecular subtyping of gastric adenocarcinoma. Hum Pathol 56: 16-21, 2016

63. Eto S, Yoshikawa K, Nishi M, Higashijima J, Tokunaga T, Nakao T, Kashihara H, Takasu C, Iwata $T$ and Shimada $M$ : Programmed cell death protein 1 expression is an independent prognostic factor in gastric cancer after curative resection. Gastric Cancer 19: 466-471, 2016.

64. Zhang L, Qiu M, Jin Y, Ji J, Li B, Wang X, Yan S, Xu R and Yang D: Programmed cell death ligand 1 (PD-L1) expression on gastric cancer and its relationship with clinicopathologic factors. Int J Clin Exp Pathol 8: 11084-11091, 2015.

65. Ohman U, Wetterfors $J$ and Moberg A: Histologic grading of gastric cancer. Acta Chir Scand 138: 384-390, 1972

c) (i) $\odot$ This work is licensed under a Creative Commons EY NC ND Attribution-NonCommercial-NoDerivatives 4.0 International (CC BY-NC-ND 4.0) License. 